\title{
METRIC DIFFERENTIABILITY OF LIPSCHITZ MAPS
}

\author{
DONATELLA BONGIORNO
}

(Received 2 August 2012; accepted 18 October 2012; first published online 15 October 2013)

Communicated by J. Borwein

\begin{abstract}
An extension of Rademacher's theorem is proved for Lipschitz mappings between Banach spaces without the Radon-Nikodým property.
\end{abstract}

2010 Mathematics subject classification: primary 26B05; secondary 49J50, 58C20, 28A15. Keywords and phrases: Lipschitz maps, Radon-Nikodým property, metric differentiability.

\section{Introduction}

Rademacher's well-known theorem states that a Lipschitz function from an open subset $G$ of $\mathbf{R}^{n}$ into $\mathbf{R}^{m}$ is Stoltz differentiable almost everywhere (Federer [9, page 216], Nekvinda and Zaijček [13], Rademacher [16]). The extension of this theorem to Lipschitz maps between infinite-dimensional Banach spaces $X$ and $Y$ has been widely investigated by various authors (Aronszajn [1], Borwein and Moors [3], Christensen [5], Mankiewicz [12], Phelps [14], Preiss and Zajíček [15] and others).

Since there is no analogue of Lebesgue measure in spaces of infinite dimension (Skorohod; see [17, page 108]), different classes of null sets were introduced to extend Rademacher's theorem. These include the Haar null sets (Christensen [4]) defined on an arbitrary Banach space (more precisely, on a complete metrizable Abelian topological group), the $\mathcal{A}$-null sets (Aronszajn [1]), the cube null sets (Mankiewicz [12]), the Gaussian null sets (Phelps [14]) and the $\widetilde{\mathcal{A}}$-null sets (Preiss and Zajičck [15]) all defined on a separable Banach space. At present the biggest class of null sets is the one introduced by Christensen, while the smallest known class of null sets is that introduced by Preiss and Zajiček. The latter is a slight modification of the Aronszajn one that is equivalent to those introduced by Mankiewicz and Phelps (Csörnyei [6]).

Supported by MURST of Italy.

(C) 2013 Australian Mathematical Publishing Association Inc. 1446-7887/2013 \$16.00 
Although the most direct extension of the Stoltz differential is the Fréchet differential, other notions of differentiability (such as Gâteaux differentiability or strict differentiability) were used to extend Rademacher's theorem since it is possible to define a Lipschitz real function that is nowhere Fréchet differentiable on each separable Banach space with nonseparable dual (see [2, Proposition 4.12]). Moreover, it was necessary to assume that the target space $Y$ satisfied the Radon-Nikodým property (Diestel and Uhl [7]), because the function $f:[0,1] \rightarrow L^{1}([0,1])$ defined by $f(t)=\chi_{[0, t]}$ is Lipschitz and nowhere Gâteaux differentiable.

Finally, Rademacher's theorem was extended (see Aronszajn [1], Mankiewicz [12], Phelps [14], Preiss and Zajíček [15] and others) for Gâteaux differentiability of Lipschitz maps from an open subset $G$ of a separable Banach space $X$ into a Banach space $Y$ with the Radon-Nikodým property; while, in the particular case where $Y \equiv \mathbf{R}$, a stronger version of Rademacher's theorem was proved (see Borwein and Moors [3]) for strict differentiability of locally Lipschitz functions defined on an open subset of a separable Banach space $X$. Here the null sets are the ones introduced by Christensen.

We recall the following facts:

- $\quad$ A mapping $f: X \rightarrow \mathbf{R}$ is said to be strictly differentiable at $x \in X$ provided that the limit

$$
\lim _{\substack{z \rightarrow x \\ t \rightarrow 0^{+}}} \frac{f(z+t u)-f(z)}{t}
$$

exists for each direction $u \in X$.

- $\quad$ A mapping $f: X \rightarrow Y$ is said to be Gâteaux differentiable at $x \in X$ provided that the limit

$$
d f_{x}(u)=\lim _{t \rightarrow 0} \frac{f(x+t u)-f(x)}{t}
$$

exists for each $u \in X \backslash\{0\}$ and $d f_{x}(\cdot)$ is a bounded linear operator.

On the other hand, when $Y$ is an arbitrary Banach space, Kirchheim proved (see [11]) that if $f: \mathbf{R}^{n} \rightarrow Y$ is Lipschitz, then the limit of the norm

$$
{ }_{m} d f_{x}(u)=\lim _{t \rightarrow 0}\left\|\frac{f(x+t u)-f(x)}{t}\right\|
$$

exists for all $u \in X \backslash\{0\}$ at almost all points $x \in \mathbf{R}^{n}$ and ${ }_{m} d f_{x}(\cdot)$ is a continuous seminorm. The number ${ }_{m} d f_{x}(u)$, when it exists, is called the metric directional derivative of $f$ at $x$ in direction $u$, and if ${ }_{m} d f_{x}(\cdot)$ is a continuous seminorm, then we say that $f$ is metrically Gâteaux differentiable at $x$.

Duda (see [8]) extended Kirchheim's result to mappings defined on an infinitedimensional separable Banach space, proving the following theorem.

Theorem 1.1. Let $X$ and $Y$ be Banach spaces. If $X$ is separable and $f: X \rightarrow Y$ is Lipschitz, then there exists an Aronszajn null set $E$ such that $f$ is metrically Gâteaux differentiable at each point $x \in X \backslash E$.

The aim of this paper is to prove that the set $E$ of Theorem 1.1 can be taken to be null in the sense of Preiss and Zajíček. This gives us a generalization of Theorem 1.1 since 
there are Aronszajn null sets that are not Preiss-Zajíček null (see [15, Proposition 13]). Moreover, since by [10, Proposition 3.5] any metric space embeds isometrically in a Banach space, our result also applies to maps with values in a metric space.

\section{Preliminaries}

Throughout this paper we let $\mathbf{R}$ denote the set of all real numbers and $\mathcal{L}(\cdot)$ denote the Lebesgue measure on $\mathbf{R}$. We assume that $X$ and $Y$ are real Banach spaces and let $B_{X}(0,1)$ denote the closed unit ball of $X$.

For each $u \in X \backslash\{0\}$ and $\varepsilon>0$ we define $\widetilde{\mathcal{A}}(u, \varepsilon)$ to be the family of all Borel sets $E \subseteq X$ with

$$
\mathcal{L}\{t \in \mathbf{R}: \varphi(t) \in E\}=0
$$

for all $\varphi: \mathbf{R} \rightarrow X$ such that the function $t \rightarrow \varphi(t)-t u$ has Lipschitz constant at most $\varepsilon$. We use $\widetilde{\mathcal{A}}(u)$ to denote the system of all sets $B$ such that $B=\bigcup_{k=1}^{\infty} B_{k}$ where $B_{k} \in$ $\widetilde{\mathcal{A}}\left(u, \varepsilon_{k}\right)$ for some $\varepsilon_{k}>0$. Moreover, if $\left\{u_{n}\right\} \subset X$ is a sequence of nonzero elements, we define

$$
\widetilde{\mathcal{A}}\left(\left\{u_{n}\right\}\right)=\left\{E \in X: E=\bigcup_{n} E_{n}\right\}
$$

where each $E_{n} \in \widetilde{\mathcal{A}}\left(u_{n}\right)$.

The Preiss-Zajíček family of null sets $\widetilde{\mathcal{A}}$ is defined to be the intersection of the families $\widetilde{\mathcal{A}}\left(\left\{u_{n}\right\}\right)$ over all possible complete sequences $\left\{u_{n}\right\}$ of $X$, that is, over all possible sequences $\left\{u_{n}\right\}$ of nonzero elements of $X$ whose linear span is dense in $X$.

Definition 2.1. Let $M$ be a subset of $X$ and let $x$ be a point of $X$. Then:

(i) $\quad M$ is said to be porous at $x$ if there exists a constant $c>0$ such that for each $\varepsilon>0$ there exists $y \in X$ with $\|y-x\|<\varepsilon$ and $0<r<c\|y-x\|$ with $M \cap B(y, r)=\emptyset$;

(ii) $\quad M$ is said to be porous at $x$ in the direction $v$ if it is porous at $x$, and $y$ satisfying the conditions of (i) can be always found having the form $y=x+t v$ where $t \geq 0$;

(iii) $M$ is said to be directionally porous at $x$ if there exists $v \in X$ such that $M$ is porous at $x$ in direction $v$;

(iv) $M$ is said to be porous (respectively, porous in the direction $v$, directionally porous) if it is porous (respectively, porous in the direction $v$, directionally porous) at each of its points;

(v) $M$ is said to be $\sigma$-porous (respectively, $\sigma$-porous in the direction $v, \sigma$ directionally porous) if it is a countable union of porous sets (respectively, sets porous in the direction $v$, directionally porous sets).

Let $G$ be an open subset of $X$ and $f: G \rightarrow Y$. For any $u, v \in X \backslash\{0\}, w, z \geq 0$ and $\varepsilon, \delta>0$ we let $A(u, v ; w, z ; \varepsilon, \delta)$ denote the set of all $x \in G$ such that:

(j) $\quad|\|f(x+t u)-f(x)\|-| t|w| \leq \varepsilon|t|$ and

$|\|f(x+t v)-f(x)\|-| t|z| \leq \varepsilon|t|$ for all $|t|<\delta$;

and

(jj) $\quad|\| f(x+t(u+v))-f(x)||-| t|(w+z)|>3 \varepsilon|t|$

for arbitrarily small $|t|$. 
Given a subset $U$ of $X \backslash\{0\}$ we define $A_{U}$ to be the union of all sets $A(u, v ; w, z ; \varepsilon, \delta)$ obtained by choosing $u, v$ in $U$, rational numbers $w, z \geq 0$ and $\varepsilon, \delta>0$.

Definition 2.2. We say that $f$ is metrically Gâteaux differentiable at $x$ if the limit

$$
{ }_{m} d f_{x}(u)=\lim _{t \rightarrow 0} \frac{\|f(x+t u)-f(x)\|}{|t|}
$$

exists for all $u \in X \backslash\{0\}$ and ${ }_{m} d f_{x}(\cdot)$ is a continuous seminorm.

Lemma 2.3. Let $U=\{u, v\}$ where $u, v \in X \backslash\{0\}$. If $x \in X \backslash A_{U}$ and the metric directional derivatives ${ }_{m} d f_{x}(u)$ and ${ }_{m} d f_{x}(v)$ exist, then ${ }_{m} d f_{x}(u+v)$ also exists and satisfies

$$
{ }_{m} d f_{x}(u+v) \leq{ }_{m} d f_{x}(u)+{ }_{m} d f_{x}(v) .
$$

Proof. Given a rational $\varepsilon>0$ there are rational numbers $w, z \geq 0$ and $\delta>0$ such that

$$
\left|w-{ }_{m} d f_{x}(u)\right|<\varepsilon / 2, \quad\left|z-{ }_{m} d f_{x}(v)\right|<\varepsilon / 2,
$$

and

$$
\begin{aligned}
& \left.|\|f(x+t u)-f(x)\|-| t\right|_{m} d f_{x}(u)|\leq \varepsilon| t \mid / 2, \\
& \left.|||f(x+t v)-f(x) \|-| t\right|_{m} d f_{x}(v)|\leq \varepsilon| t \mid / 2,
\end{aligned}
$$

for all $|t|<\delta$. It follows from (2.1) and (2.2) that

$$
\begin{gathered}
|||f(x+t u)-f(x) \|-| t|w| \leq \varepsilon|t| / 2+|t|\left|w-{ }_{m} d f_{x}(u)\right|<|t| \varepsilon \\
\quad|\|f(x+t v)-f(x)\|-| t|z| \leq \varepsilon|t| / 2+|t|\left|z-{ }_{m} d f_{x}(u)\right|<|t| \varepsilon
\end{gathered}
$$

for all $|t|<\delta$.

Now since $x \notin A(u, v ; w, z ; \varepsilon, \delta)$ and (j) holds, there exists $\eta>0$ such that

$$
|\|f(x+t(u+v))-f(x)\|-| t|(w+z)| \leq 3 \varepsilon|t|
$$

for all $0<|t|<\eta$. Consequently,

$$
\begin{aligned}
& ||\left|\frac{f(x+t(u+v))-f(x)}{t}\|-\| \frac{f(x+s(u+v))-f(x)}{s} \|\right| \mid \\
& \leq||\left|\frac{f(x+t(u+v))-f(x)}{t} \|-\right| t|(w+z)| \\
& \quad+|| \frac{f(x+s(u+v))-f(x)}{s} \|-|t|(w+z) \mid \\
& \leq 6 \varepsilon
\end{aligned}
$$

for all $0<|t|,|s|<\eta$. By Cauchy's criterion this implies the existence of the metric directional derivative ${ }_{m} d f_{x}(u+v)$. Moreover, by (2.1) and (2.3),

$$
{ }_{m} d f_{x}(u+v) \leq 3 \varepsilon+(y+z) \leq 4 \varepsilon+{ }_{m} d f_{x}(u)+{ }_{m} d f_{x}(v) .
$$


Hence, since $\varepsilon$ is arbitrary,

$$
{ }_{m} d f_{x}(u+v) \leq{ }_{m} d f_{x}(u)+{ }_{m} d f_{x}(v)
$$

as required.

Lemma 2.4. If $f$ is Lipschitz, then for each countable dense subset $U$ of $X \backslash\{0\}$ the set $A_{U}$ is $\sigma$-directionally porous.

Proof. By definition of $A_{U}$ it is enough to prove that for $u$ and $v$ in $U$, rational numbers $w, z \geq 0$ and $\varepsilon, \delta>0$, the set $A(u, v ; w, z ; \varepsilon, \delta)$ is porous at each of its points in the direction $v$.

Let $x \in A(u, v ; w, z ; \varepsilon, \delta)$, and define $c=\frac{1}{2} \operatorname{Lip}(f)$ where $\operatorname{Lip}(f)$ is the Lipschitz constant of $f$. For given $0<\varepsilon^{\prime}<2 \varepsilon$ let $t \in \mathbf{R}$ satisfy $\|t v\|<\varepsilon^{\prime}$ and define $y=x+t v$, $r=c\|t v\| / 2$. Take $q \in B(y, r)$ and assume that $q \in A(u, v ; w, z ; \varepsilon, \delta)$. Then, by the definition of $A(u, v ; w, z ; \varepsilon, \delta)$,

$$
\begin{aligned}
&\|f(q+t u)-f(q)\|-|t| w \geq \| f(x+t(u+v))-f(x+t v) \|-|t| w \\
&-2 \operatorname{Lip}(f)\|q-(x+t v)\| \\
&>\|f(x+t(u+v))-f(x)\|-|t|(w+z) \\
& \quad-(\|f(x+t v)-f(x)\|-|t| z)-\frac{\varepsilon^{\prime}|t|}{2} \\
&>\varepsilon|t| .
\end{aligned}
$$

But this contradicts $(\mathrm{j})$. Therefore $q \notin A(u, v ; w, z ; \varepsilon, \delta)$, as required.

Lemma 2.5. Let $f$ be Lipschitz and let $x \in G$. If the metric directional derivative ${ }_{m} d f_{x}(u)$ exists in all directions $u$ from a set $U_{x}$ whose linear span is dense in $X$, then the metric directional derivative ${ }_{m} d f_{x}(v)$ exists for each $v \in X \backslash\{0\}$ and

$$
\left|{ }_{m} d f_{x}(u)-{ }_{m} d f_{x}(v)\right| \leq \operatorname{Lip}(f)\|u-v\|
$$

for each $u, v \in X \backslash\{0\}$.

Proof. By Lemma 2.3 the metric directional derivative ${ }_{m} d f_{x}(u)$ exists in all directions $u$ from the linear span $V_{x}$ of $U_{x}$. Now let $v \in X \backslash\{0\}$. Since $V_{x}$ is dense in $X$, given $\varepsilon>0$ we can find $u \in V_{x}$ such that

$$
\|u-v\|<\frac{\varepsilon}{4} \operatorname{Lip}(f) .
$$

Moreover, by the existence of ${ }_{m} d f_{x}(u)$, there is $\delta>0$ such that

$$
|| \frac{f(x+t u)-f(x)}{t}\|-\| \frac{f(x+s u)-f(x)}{s} \| \mid<\varepsilon / 2
$$

for each $0<|t|,|s|<\delta$. 
Now since

$$
\begin{aligned}
& \left\|\frac{f(x+t v)-f(x)}{t}\right\| \leq\left\|\frac{f(x+t u)-f(x)}{t}\right\|+\operatorname{Lip}(f)\|u-v\|, \\
& \left\|\frac{f(x+s u)-f(x)}{s}\right\| \leq\left\|\frac{f(x+s v)-f(x)}{s}\right\|+\operatorname{Lip}(f)\|u-v\|,
\end{aligned}
$$

it follows that

$$
\begin{aligned}
& \left\|\frac{f(x+t v)-f(x)}{t}\right\|-\left\|\frac{f(x+s v)-f(x)}{s}\right\| \\
& \leq\left\|\frac{f(x+t u)-f(x)}{t}\right\|-\left\|\frac{f(x+s u)-f(x)}{s}\right\|+2 \cdot \operatorname{Lip}(f)\|u-v\|<\varepsilon
\end{aligned}
$$

for each $0<|t|,|s|<\delta$. Exchanging $s$ and $t$ gives

$$
\left\|\left|\left\|\frac{f(x+t v)-f(x)}{t}\right\|-\left\|\frac{f(x+s v)-f(x)}{s}\right\|\right|<\varepsilon\right.
$$

for each $0<|t|,|s|<\delta$. So, by Cauchy's criterion, the metric directional derivative ${ }_{m} d f_{x}(v)$ exists.

Finally, condition (2.4) is a simple consequence of the inequality

$$
\|\| \frac{f(x+t u)-f(x)}{t}\|-\| \frac{f(x+t v)-f(x)}{t}\|\mid \leq\| \frac{f(x+t u)-f(x+t v)}{t}\|\leq \operatorname{Lip}(f)\| u-v \|
$$

and our result is established.

THEOREM 2.6. If $X$ is separable and $f$ is Lipschitz, then the following implication holds at each point $x \in G$ outside a $\sigma$-directionally porous set.

- If ${ }_{m} d f_{x}(u)$ exists in all directions $u$ from a set $U_{x}$ whose linear span is dense in $X$, then $f$ is metrically Gâteaux differentiable at $x$.

Proof. The result is trivial if $f$ is a constant mapping, so we may assume that $\operatorname{Lip}(f)>0$. Let $G^{\#}$ be the set of all $x \in G$ at which the metric directional derivative ${ }_{m} d f_{x}(u)$ exists in all directions $u$ from a set $U_{x}$ whose linear span is dense in $X$. Since $X$ is separable, we can assume that $U_{x}$ is countable. Let $V_{x}$ be the linear span of $U_{x}$ with rational coefficients. Then $V_{x}$ is a countable dense subset of $X$.

Next we let $G_{\#}$ be a countable dense subset of $G^{\#}$ and define $U$ to be the union of all sets $V_{x}$ obtained by choosing $x$ in $G_{\#}$. Since $U$ is a countable dense subset of $X$, the set $A_{U}$ is $\sigma$-directionally porous by Lemma 2.4. To complete the proof, it is enough to show that $f$ is metrically Gâteaux differentiable at each point of $G^{\#} \backslash A_{U}$.

Let $x \in G^{\#} \backslash A_{U}$. By Lemma 2.5 the metric directional derivative ${ }_{m} d f_{x}(u)$ exists for each $u \in X \backslash\{0\}$ and:

(i $\left.{ }_{1}\right) \quad{ }_{m} d f_{x}(u) \leq \operatorname{Lip}(f)\|u\|$ for each $u \in X \backslash\{0\}$;

(i $\left.\mathrm{i}_{2}\right) \quad{ }_{m} d f_{x}(\lambda u)=\lim _{t \rightarrow 0}\|(f(x+t \lambda u)-f(x)) / t\|$

$$
=\lim _{t \rightarrow 0}|\lambda|||(f(x+t \lambda u)-f(x)) / t \lambda \|\left.|=| \lambda\right|_{m} d f_{x}(u)
$$

for each $u \in X \backslash\{0\}$ and $\lambda \in \mathbf{R}$;

(i $\left.i_{3}\right) \quad{ }_{m} d f_{x}(u+v) \leq{ }_{m} d f_{x}(u)+{ }_{m} d f_{x}(v)$ for each $u, v \in U$ by Lemma 2.3. 
Now let $u_{0}, v_{0} \in X \backslash\{0\}$. Since $U$ is dense in $X$, for each $\varepsilon>0$ there are $u, v \in U$ such that $\left\|u-u_{0}\right\|<\varepsilon$ and $\left\|v-v_{0}\right\|<\varepsilon$. Therefore, by (2.4) and $\left(\mathrm{i}_{3}\right)$,

$$
\begin{aligned}
{ }_{m} d f_{x}\left(u_{0}+v_{0}\right) & \leq \operatorname{Lip}(f)\left(\left\|u-u_{0}\right\|+\left\|v-v_{0}\right\|\right)+{ }_{m} d f_{x}(u+v) \\
& \leq 2 \varepsilon \operatorname{Lip}(f)+{ }_{m} d f_{x}(u)+{ }_{m} d f_{x}(v) \\
& \leq 4 \varepsilon \operatorname{Lip}(f)+{ }_{m} d f_{x}\left(u_{0}\right)+{ }_{m} d f_{x}\left(v_{0}\right) .
\end{aligned}
$$

Hence, since $\varepsilon$ was chosen arbitrarily, it follows that

$$
{ }_{m} d f_{x}\left(u_{0}+v_{0}\right) \leq{ }_{m} d f_{x}\left(u_{0}\right)+{ }_{m} d f_{x}\left(v_{0}\right) .
$$

Moreover, ( $\left.\mathrm{i}_{1}\right)$ implies that ${ }_{m} d f_{x}(\cdot)$ is a continuous seminorm. So $f$ is metrically Gâteaux differentiable at $x$ and our result is established.

\section{Rademacher's theorem in generic Banach spaces}

As in the previous section, we assume that $G$ is an open subset of $X$. Let $\left\{u_{n}\right\} \subset X$ and let $h: X \rightarrow \mathbf{R}$. We define $\Delta$ to be the set of all $x \in G$ at which $h$ is not metrically Gâteaux differentiable. For $n=1,2, \ldots$, we define $\Delta_{u_{n}}$ to be the set of all $x \in G$ for which ${ }_{m} d h_{x}\left(u_{n}\right)$ does not exist and $\Delta_{u_{n}}^{h}$ to be the set of all $x \in G$ for which $d h_{x}\left(u_{n}\right)$ does not exist.

The next theorem is an extension of [15, Theorem 10] to metrically Gâteaux differentiable Lipschitz mappings. Although the proof is the same, apart from using Theorem 2.6 instead of [15, Theorem 2], we include it for completeness.

THEOREM 3.1. If $X$ is separable and $f: G \subseteq X \rightarrow Y$ is Lipschitz, then for every complete sequence $\left\{u_{n}\right\}$ in $X$ the set $\Delta$ belongs to the Borel $\sigma$-ideal generated by the sets of the form $\Delta_{u_{n}}, n=1,2, \ldots$, and $\Delta_{u_{n}}^{h}, n=1,2, \ldots$, where $h: X \rightarrow \mathbf{R}$ is Lipschitz.

Proof. Let $N=\Delta \backslash \bigcup_{n} \Delta_{u_{n}}$. By Theorem 2.6, $N$ is $\sigma$-directionally porous and so by [15, Theorem 1] we can write $N=\bigcup_{n=1}^{\infty}\left(N_{n}^{+} \cup N_{n}^{-}\right)$where $N_{n}^{+}$and $N_{n}^{-}$are Borel sets which are $\sigma$-porous in directions $u_{n}$ and $-u_{n}$, respectively. Let $N_{n, k}^{+}$and $N_{n, k}^{-}$be sets which are porous in directions $u_{n}$ and $-u_{n}$, respectively and such that $N_{n}^{+}=\bigcup_{n=1}^{\infty} N_{n, k}^{+}$ and $N_{n}^{-}=\bigcup_{n=1}^{\infty} N_{n, k}^{-}$.

The functions $\phi_{n, k}(x)=\operatorname{dist}\left(x, N_{n, k}^{+}\right)$and $\psi_{n, k}(x)=\operatorname{dist}\left(x, N_{n, k}^{-}\right)$are real-valued Lipschitz functions on $X$. Moreover, $N_{n, k}^{+}$is a proper subset of the set of all $x \in X$ such that $d\left(\phi_{n, k}\right)_{x}\left(u_{n}\right)$ does not exist and $N_{n, k}^{-}$is a proper subset of the set of all $x \in X$ such that $d\left(\psi_{n, k}\right)_{x}\left(u_{n}\right)$ does not exist. Our result follows.

We now extend ideas from [15, Lemma 11] to metrically Gâteaux differentiable Lipschitz mappings. For $x \in G, u \in X \backslash\{0\}$ and $\varepsilon>0$ we set

$$
g^{\varepsilon}(f, x, u)=\sup _{0<|t|,|s| \leq \varepsilon}\left|\left\|\frac{f(x+t u)-f(x)}{t}\right\|-\left\|\frac{f(x+s u)-f(x)}{s}\right\|\right|
$$

and

$$
g(f, x, u)=\lim _{\varepsilon \rightarrow 0^{+}} g^{\varepsilon}(f, x, u) .
$$


Lemma 3.2. For each $u \in X \backslash\{0\}$ and $c \geq 0$ the set

$$
G_{c}=\{x \in G: g(f, x, u)>c\}
$$

is Borel.

Proof. For $h, k \in \mathbf{N}$ we set

$$
G_{c}^{h, k}=\left\{x \in G: g^{1 / k}(f, x, u)>c+\frac{1}{h}\right\} .
$$

It is easy to check that

$$
G_{c}=\bigcup_{h=1}^{\infty} \bigcap_{k=1}^{\infty} G_{c}^{h, k}
$$

We prove that $G_{c}^{h, k}$ is an open set. It will then follow that $G_{c}$ is a $G_{\delta \sigma}$ set.

If $x \in G_{c}^{h, k}$ there is $0<\left|t_{0}\right|,\left|s_{0}\right| \leq 1 / k$ such that

$$
\left|\left\|\frac{f\left(x+t_{0} u\right)-f(x)}{t_{0}}\right\|-\left\|\frac{f\left(x+s_{0} u\right)-f(x)}{s_{0}}\right\|\right|>c+\frac{1}{h} .
$$

Since the function

$$
y \rightarrow\left|\left\|\frac{f\left(y+t_{0} u\right)-f(y)}{t_{0}}\right\|-\left\|\frac{f\left(y+s_{0} u\right)-f(y)}{s_{0}}\right\|\right|
$$

is continuous, there exists $r>0$ such that

$$
\left|\left\|\frac{f\left(y+t_{0} u\right)-f(y)}{t_{0}}\right\|-\left\|\frac{f\left(y+s_{0} u\right)-f(y)}{s_{0}}\right\|\right|>c+\frac{1}{h}
$$

for each $y \in B(x, r)$. This implies that $B(x, r) \subset G_{c}^{h, k}$, that is, $G_{c}^{h, k}$ is an open set, as required.

The following lemma is an immediate consequence of our definitions.

Lemma 3.3. If $f: G \rightarrow Y$ is Lipschitz, then the metric directional derivative ${ }_{m} d f_{x}(u)$ exists if and only if $g(f, x, u)=0$.

Lemma 3.4. Let $f: G \rightarrow Y$ be Lipschitz, $u \in X \backslash\{0\}, r \in \mathbf{R}$ and assume that there exists $\varphi: \mathbf{R} \rightarrow X$ such that:

(i) $\quad \psi: t \rightarrow \varphi(t)-t u$ is Lipschitz;

(ii) $\operatorname{Lip}(f) \cdot \operatorname{Lip}(\psi)<g(f, \varphi(r), u) / 4$.

Then the mapping $f \circ \varphi$ is not metrically Gâteaux differentiable at the point $r$.

Proof. By Lemma 3.3 it is enough to prove that $g(f \circ \varphi, r, 1)>0$. Define $x=\varphi(r)$. By the definition of $g(f, x, u)$ it follows that for any given $\varepsilon>0$ we can find $0<|t|,|s|<\varepsilon$ such that

$$
\left\|\frac{f(x+t u)-f(x)}{t}-\frac{f(x+s u)-f(x)}{s}\right\|>\frac{3}{4} g(f, x, u) .
$$


Then using (i) and (ii) we calculate

$$
\begin{aligned}
\left\|\frac{f(x+t u)-f \circ \varphi(r+t)}{t}\right\| & \leq \operatorname{Lip}(f)\left\|\frac{\varphi(r)+t u-\varphi(r+t)}{t}\right\| \\
& =\operatorname{Lip}(f)\left\|\frac{\psi(r)-\psi(r+t)}{t}\right\| \\
& \leq \operatorname{Lip}(f) \cdot \operatorname{Lip}(\psi)<\frac{g(f, \varphi(r), u)}{4} .
\end{aligned}
$$

By a similar argument we may deduce that

$$
\left\|\frac{f(x+s u)-f \circ \varphi(r+s)}{s}\right\|<\frac{g(f, \varphi(r), u)}{4} .
$$

Consequently,

$$
\begin{aligned}
&\left\|\frac{f \circ \varphi(r+t)-f \circ \varphi(r)}{t}-\frac{f \circ \varphi(r+s)-f \circ \varphi(r)}{s}\right\| \\
& \geq\left\|\frac{f(x+t u)-f(x)}{t}-\frac{f(x+s u)-f(x)}{s}\right\| \\
&-\left\|\frac{f(x+t u)-f \circ \varphi(r+t)}{t}\right\| \\
&-\left\|\frac{f(x+s u)-f \circ \varphi(r+s)}{s}\right\| \\
&> \frac{g(f, \varphi(r), u)}{4} .
\end{aligned}
$$

So we may deduce that

$$
g(f \circ \varphi, r, 1) \geq \frac{g(f, \varphi(r), u)}{4}>\operatorname{Lip}(f) \cdot \operatorname{Lip}(\psi) \geq 0
$$

as required.

Theorem 3.5. If $X$ is separable, $G \subseteq X$ is open and $f: G \rightarrow Y$ is Lipschitz, then the set $\Delta$ of points $x \in G$ at which $f$ is not metrically Gâteaux differentiable belongs to $\widetilde{\mathcal{A}}$.

Proof. Let $\left\{u_{n}\right\}$ be a complete sequence in $X$. We need to prove that $\Delta \in \widetilde{\mathcal{A}}\left(\left\{u_{n}\right\}\right)$. By Theorem 3.1, $\Delta$ belongs to the Borel $\sigma$-ideal generated by the sets of the forms $\Delta_{u_{n}}$ and $\Delta_{u_{n}}^{h}$ where $h: X \rightarrow \mathbf{R}$ is Lipschitz and $n=1,2, \ldots$ Therefore, since $\Delta_{u_{n}}^{h} \in \widetilde{\mathcal{A}}\left(u_{n}\right)$ by [15, Theorem 12], it is enough to prove that $\Delta_{u} \in \widetilde{\mathcal{A}}(u)$ for each $u \in X \backslash\{0\}$.

We define

$$
G_{k}=\left\{x \in G: g(f, x, u)>\frac{\operatorname{Lip}(f)}{k}\right\}, \quad k \in \mathbf{N} .
$$

By Lemma 3.2, the sets $G_{k}$ are Borel sets, and by Lemma 3.3,

$$
\Delta_{u}=\bigcup_{k=1}^{\infty} G_{k} .
$$


Let $\varphi: \mathbf{R} \rightarrow X$ be such that the function $\psi: t \rightarrow \varphi(t)-t u$ is Lipschitz and Lip $(\psi) \leq$ $1 / 4 k$. If $t \in \mathbf{R}$ is such that $\varphi(t) \in G_{k}$, then

$$
g(f, \varphi(t), u)>\operatorname{Lip}(f) / k \geq 4 \operatorname{Lip}(f) \operatorname{Lip}(\psi)
$$

and so, by Lemma 3.4, the function $f \circ \varphi$ is not metrically Gâteaux differentiable at $t$. Hence

$$
\left\{t \in \mathbf{R}: \varphi(t) \in G_{k}\right\}
$$

is a subset of the set of all points $t \in \mathbf{R}$ at which $f \circ \varphi$ is not metrically Gâteaux differentiable.

Now $f \circ \varphi$ is a Lipschitz map from $\mathbf{R}$ into $Y$. It follows by [11, Theorem 3.5] that

$$
\mathcal{L}\left\{t \in \mathbf{R}: \varphi(t) \in G_{k}\right\}=0 .
$$

Hence $G_{k} \in \widetilde{\mathcal{A}}(u, 1 / 4 k)$ and consequently $\Delta_{u} \in \widetilde{\mathcal{A}}(u)$.

\section{Extension to metric spaces}

Let $(S, d)$ be a metric space and let $f: G \rightarrow S$ where, as usual, $G$ is an open subset of a Banach space $X$. We say that $f$ is Gâteaux differentiable at $x \in G$ if the limit

$$
{ }_{m} d f_{x}(u)=\lim _{t \rightarrow 0} \frac{d(f(x+t u), f(x))}{|t|}
$$

exists for all $u \in X \backslash\{0\}$ and ${ }_{m} d f_{x}(\cdot)$ is a continuous seminorm.

THeOREM 4.1. If $X$ is a separable Banach space, $(S, d)$ is a metric space and $f: G \rightarrow S$ is Lipschitz, then the set $\Delta$ of points $x \in G$ at which $f$ is not Gâteaux differentiable belongs to $\widetilde{\mathcal{A}}$.

Proof. By [10, Proposition 3.5] there exist a compact Hausdorff space $K$ and an isometry $T:(S, d) \rightarrow\left(C(K),\|\cdot\|_{\infty}\right)$. The mapping $\tilde{f}=T \circ f$ is a Lipschitz mapping from $G$ into the Banach space $\left(C(K),\|\cdot\|_{\infty}\right)$. So, by Theorem $3.5, \tilde{f}$ is metrically Gâteaux differentiable outside a set $\Delta$ belonging to $\widetilde{\mathcal{A}}$.

Moreover, for each $x \in G, u \in X \backslash\{0\}$ and $t \neq 0$,

$$
\frac{d(f(x+t u), f(x))}{|t|}=\left\|\frac{\tilde{f}(x+t u)-\tilde{f}(x)}{t}\right\|_{\infty} .
$$

Thus, if $x \in G \backslash \Delta$, the directional derivative ${ }_{m} d f_{x}(u)$ exists and ${ }_{m} d f_{x}(u)={ }_{m} d \tilde{f}_{x}(u)$. This implies that ${ }_{m} d f_{x}(\cdot)$ is a continuous seminorm; hence $f$ is Gâteaux differentiable at $x$. 


\section{References}

[1] N. Aronszajn, 'Differentiability of Lipschitzian mappings between Banach spaces', Stud. Math. 57 (1976), 147-190.

[2] Y. Benyamini and J. Lindenstrauss, Geometric Nonlinear Functional Analysis, Colloquium Publications, 48 (American Mathematical Society, Providence, RI, 2000).

[3] J. M. Borwein and W. B. Moors, 'Null sets and essentially smooth Lipschitz functions', SIAM J. Optim. 8 (1998), 309-323.

[4] J. P. R. Christensen, 'On sets of Haar measure zero in Abelian groups', Israel. J. Math. 13 (1972), 255-260.

[5] J. P. R. Christensen, 'Measure theoretic zero sets in infinite dimensional spaces and applications to differentiability of Lipschitz mappings', Publ. Dépt. Math. Lyon 10(2) (1973), 29-39.

[6] M. Csörnyei, 'Aronszajn null and Gaussian null sets coincide', Israel J. Math. 111 (1999), 191201.

[7] J. Diestel and J. J. Uhl Jr., Vector Measures, Mathematical Surveys, 15 (American Mathematical Society, Providence, RI, 1977).

[8] J. Duda, 'Metric and $w^{*}$-differentiability of pointwise Lipschitz mappings', Z. Anal. Anwend. 26 (2007), 341-362.

[9] H. Federer, Geometric Measure Theory (Springer, Berlin, 1969).

[10] P. S. Kenderov and W. B. Moors, 'Fragmentability of groups and metric-valued function spaces', Topology Appl. 159 (2012), 183-193.

[11] B. Kirchheim, 'Rectifiable metric spaces: local structure and regularity of the Hausdorff measure', Proc. Amer. Math. Soc. 121 (1994), 113-123.

[12] P. Mankiewicz, 'On the differentiability of Lipschitz mappings in Fréchet spaces', Studia Math. 45 (1973), 15-29.

[13] A. Nekvinda and L. Zajíček, 'A simple proof of the Rademacher theorem', Časopis Pěst. Mat. 113 (1988), 337-341.

[14] R. Phelps, 'Gaussian null sets and differentiability of Lipschitz maps on Banach spaces', Pacific J. Math. 77 (1978), 523-531.

[15] D. Preiss and L. Zajíček, 'Directional derivatives of Lipschitz functions', Israel J. Math. 125 (2001), 1-27.

[16] H. Rademacher, 'Über partielle und totale Differenzierbarkeit I', Math. Ann. 79 (1919), 254-269.

[17] A. V. Skorohod, Integration in Hilbert spaces, Ergebnisse der Mathematik und ihrer Grenzgebiete, 79 (Springer, New York, 1974).

DONATELLA BONGIORNO, Università degli studi di Palermo, Dipartimento dell'Energia, Ingegneria dell'Informazione e Modelli Matematici (DEIM), Viale delle Scienze Ed. 9, 90128 Palermo, Italy e-mail: donatella.bongiorno@unipa.it 